\title{
Diagrams of Intensity: Visualising the Sensibilities of Transdisciplinary Practice
}

Profiling Emerging Research Innovations

\author{
Romaine Logere \\ RMIT University School of Media and Communication, Melbourne, \\ Victoria, Australia \\ romaine.logere@gmail.com
}

\begin{abstract}
One of the challenges to an increased rationalism within educational discourse has been a rethinking of mind-body relations. While there has been considerable discussion around what is implicated through the engagement of physical and theoretical sites of knowing, methodological difficulties related to how its resultant data might be meaningfully evidenced remain. Based on fieldwork conducted on a postqualitative approach to transdisciplinary practice the author provides an account of a visual research method developed specifically to illustrate non-verbal experiences of group ideation. Writing from the position of a creative practitioner and intimate insider, the author explores how this positionality supported the role of bodily knowing in her research and the ways in which bodily experience offered utility to this research endeavour. The author concludes with a reflection on visualisation as a method to capture non-cognitive data and areas indicated through felt data for further exploration.
\end{abstract}

\section{Keywords}

emerging research innovations - transdisciplinarity - bodily knowing - visual research - diagrams 
- This article is part of the special topic 'Profiling Emerging Research Innovations', edited by Elaine Khoo.

- Strand:Visual methodology in educational research - new practices

- Topic: Applications of visual or video methods as a methodology, as a tool for data collection, analysis and representation of findings.

\section{$1 \quad$ Introduction}

This article examines methodological challenges presented by pursuing sites of physical and theoretical knowing in research. While there has been increasing moves away from the traditional mind-body hierarchies in relation to knowledge, questions remain regarding what foregrounding the physical in research enables and how an "evidentiary warrant" (St. Pierre, 1997, p. 179) might exist for data that is resistant to documentation. On the basis of my recent fieldwork experiences researching transdisciplinary practice, I explore these two dilemmas as they pertain to practitioners who work intentionally with "response data" (St. Pierre, 1997, p. 179).

Claims to a transdisciplinary practice are often vexed due to the terminological confusion that still surrounds the term. Recently it has been submitted that multiple definitions and methodologies could reasonably be attributed to its practice (Klein, 2013). For the purpose of this discussion the transdisciplinary practice referred to in this article is founded in what is known as the Nicolescuian approach. Based on the principles espoused by theoretical physicist and transdisciplinary scholar Basarab Nicolescu, there are a number of tenets central to this form of transdisciplinarity which distinguish it from other modes of practice. Most pertinent to this inquiry is its recognition of other systems of knowledge. Specifically, those borne from sensibility and the body (see de Freitas, et al., 1994).

Physicality as a fundamental contributor to transdisciplinary knowledge is explicitly stated or implicated across a range of salient texts (Brenner, 2004; de Freitas, et al., 1994; McGregor, 2015; Nicolescu, 2010, 2014; Winquist, 1982). In its effort to "[occasion] the emergence of new data" (de Freitas, et al., 1994, para. 11) the Nicolescuian approach extends the collaborative encounter beyond the interplay of different disciplines to include "the mobilisation of sensibility in concert with intellect" (Barnacle, 2009, p. 31). The questions to which this article now attends is what bodily knowing implicates in transdisciplinary practice and how might its data be meaningfully expressed.

Addressing the first point, an indication of the role of the body in transdisciplinary knowing is offered by education researcher, Robert Winquist (1982). Winquist correlates Michael Polanyi's (1967) concept of indwelling with 
transdisciplinary transcendence. He argues Polanyi's notion of indwelling as a physical phenomena plays a key role in the progression of extant knowledge (the example of learning to drive is provided by Polanyi as a demonstration of physical learning in combination with instruction). Winquist corresponds indwelling to non-verbal, transcendent states occasioned through transdisciplinary collaboration whereby revelatory insight(s) are assimilated within one's present knowledge. He notes this process is typically understood as requiring a period of passive and tacit incubation, adding that its absence of outward expression does not indicate of a lack of substance, but instead is indicative of its "dynamical integrative power" (1982, p. 62).

Similarly, philosopher and transdisciplinary practitioner, Erin Manning submits that the "radical shift[s]" (Manning, n.d.) she experiences in her collaborative work are first felt at a physical level and are "ineffable. But these shifts alter, at some level, what is possible" (Manning, n.d.). Manning attributes to this wordless, physical process the term "emissary" (Manning, n.d.); word with its etymological origins in "scout" or "spy", and, beyond that, from emittere, "to send out" (lexico, 2020). In this context, the knowledge the emissary passes on is not articulated. It is experienced first as a physical reflex before it can be secured through language.

While there is a growing body of work examining the significance of bodily knowing in research (Barbour, 2004; Barnacle, 2009; Pillow, 2003; St. Pierre, 1997), questions around how its data might be usefully purposed and captured remain. In this article I intend to explore how bodily knowing was engaged through an investigation of my practice and how the challenge of providing an account for its role in the research was addressed.

\section{The Present Study}

Over 2017 I spent twelve months conducting ethnographic fieldwork with a range of participants working in the university I was based in. The initial aim of my project was to investigate what creative research methods make possible in transdisciplinary practice. It was based on a practice I had run for ten years, during which five event formats were developed and run as individual event programs. Of these event series each ran over a mean period of two years. Three different event formats were selected for the investigation based upon the adaptability of their design to the research requirements:

After a preliminary investigation into a range of potential research methodologies I decided to adopt an action research design in which I would position myself as an intimate insider. This meant all the participants I engaged through the research would be drawn from a consortium of people with whom 
Event Strategy

wunderkammer Reconsidering data:

Uses metaphor to express the unquantifiable aspects of quantitative data

intertextualfilm Intertextual analysis:

night Connotative responses to a (set) research inquiry

parallel Recontextualising data:

fascinations Builds relational thresholds between dual practices

I shared a pre-established collegial history. My aim was to situate myself within the research in a way that supported an engagement with the participants physical and theoretical ways of knowing. Anthropologist John L. Aguilar's description is useful here:

Insiders ... point out that because of their greater linguistic competence they can phrase questions in a manner more comfortable and meaningful to the informants. Another claim is greater ability to read nonverbal indications of such subjective states as suspicion, confidence, and embarrassment. This enables the insider researcher to adapt his own behaviour more effectively to facilitate the flow of information. This ability to read behavioural cues, according to one individual, permits him to gauge more accurately the trustworthiness of informants' verbal responses and accounts.

AGUILAR, 1981, p. 18

It was my intention to apply the pre-existing familiarity I shared with the participants away from interactions dependent on language toward relational attunements that "listen[ed] with [the] skin" (Goldberger, 1996, p. 354). Sociologist Kelly Chong (2008) cites sociologist Meredith McGuire in her submission that making an affordance for felt experience within the investigation process allows the researcher to access:

[T] he moods of the group, the periods of fervor, the moments of awe ... these feelings which one experiences are not merely subjective reactions but empirical data; it is the researcher's task to clarify what aspects of the experience account for these feelings.

CHONG, 2008, p. 378 citing McGuire, 1982 
My fieldwork involved participant observation, reflective journaling, interviewing and an intensive transcription and coding process that occurred over repeated intervals. Just over half the participants involved identified as interor transdisciplinary or as having had some experience of working in inter- or transdisciplinary ways. With the exception of one participant, all worked in the university sector and were at various points in their academic career. The participant selection represented a range of disciplinary backgrounds. Respectively these were: sociology, anthropology, graphic design, political science, architecture, film and creative practice.

While a number of participants contributed to this research, for this article I have focussed on my experiences with the participants I have identified through the pseudonyms Jan and Gerda. Jan was a colleague I had known for over a decade. He had presented and been a regular attendee at the first transdisciplinary events I co-ran and was therefore well acquainted with my approach. He had been employed by the university for the entire time I had known him and was a familiar face within the university's School of Art, and School of Architecture and Urban Design. He had a research practice and wide-ranging experience in the field of socially engaged art and design. The second participant featured in this article is referred to as Gerda. At the time of my research, Gerda was a Vice Chancellor's Research Fellow. We had met years earlier when I had been working in research communications at a transdisciplinary institute, with whom she was likewise employed. While her background was in sociology, Gerda variously identified herself as a trans- or interdisciplinary practitioner. The focus of her research was on spatial experience, which she complemented with design research and visual methodologies.

Jan was one of the participants who attended the first event, wunderkammer, run as part of my investigation on my practice. In its original form wunderkammer ran from 2008-2010. The intention of this event was to challenge a group of creative practitioners to find metaphoric ways of expressing quantitative data. It was called wunderkammer with the idea that, like its namesake, a collection of 'curious' research objects would be produced as a result of its endeavours. For the version of this event recomposed for my study I invited a political science doctoral candidate I had struck up a friendship with to present her research to a group of creatives. The creatives came from a range of disciplinary backgrounds, including architecture, film and creative practice. The creative participants were tasked with assisting the political science candidate communicate what she described as the unsaid, by which she meant conspicuous absences of information in environmental policy. The discussions that ensued were very lively and the group quickly charged off across a range of conversational topics. 
Early in the research I had made the decision that I would document what occurred in the events with audio recordings, written transcriptions and photographs. Further, that I would transcribe the discussions and post-event participant interviews myself. My rationalization was that my participation in the interactions would provide valuable context to the audio recording, and therefore minimalize inaccuracies in the transcriptions. However, while transcribing the recordings did helpfully revisit the interactions and the recollections I had of what had occurred during the exchanges, it became increasingly clear there were moments I recalled or heard that were going to be impossible to transcribe. These points appeared to have a pattern and largely presented as variations of the following: Either the recording would pick up just the sound of physical movement (the sounds of pens and sleeves moving across paper; chairs legs squeaking as participants moved, etc.), or the interaction consisted of non-linguistic vocal exchanges (grunts, laughter, etc.). Frustratingly, I realised these moments were often occurring at peak points in group cohesion as the participants were progressing toward a shared insight. Listening to the sounds of the physical movements prompted a felt recollection of what was occurring, but although I experienced an embodied sense of clarity of what had transpired, it was a phenomenon difficult to transcribe into words. Reviewing the photographs I had taken of the event's proceedings to see if this form of documentation was able to visually capture the group's momentum revealed yet further unanticipated challenges my research faced (see figures 1 \& 2 ).

In the first case, it became clear that while positioning myself as an intimate insider assisted my understanding of the group's felt experience, I would have to suspend the intimacy of that role during the events and switch to a more detached observer position if I wanted comprehensive documentation of the group's interactions. Further, that audio and visual recordings as documentation methods were going to be inherently ineffective in this context of the research. The photos could provide a visual account of moments in the group interaction. The audio recording was able to relay the spoken elements of group interaction. But the felt experience of knowledge was fundamentally resistant to both these forms of documentation.

These realisations were then further underscored when I shared this dilemma with the participant Jan during a post-event interview. In response to remarks I made on the difficulties I was encountering in transcribing these non-linguistic exchanges he replied: 


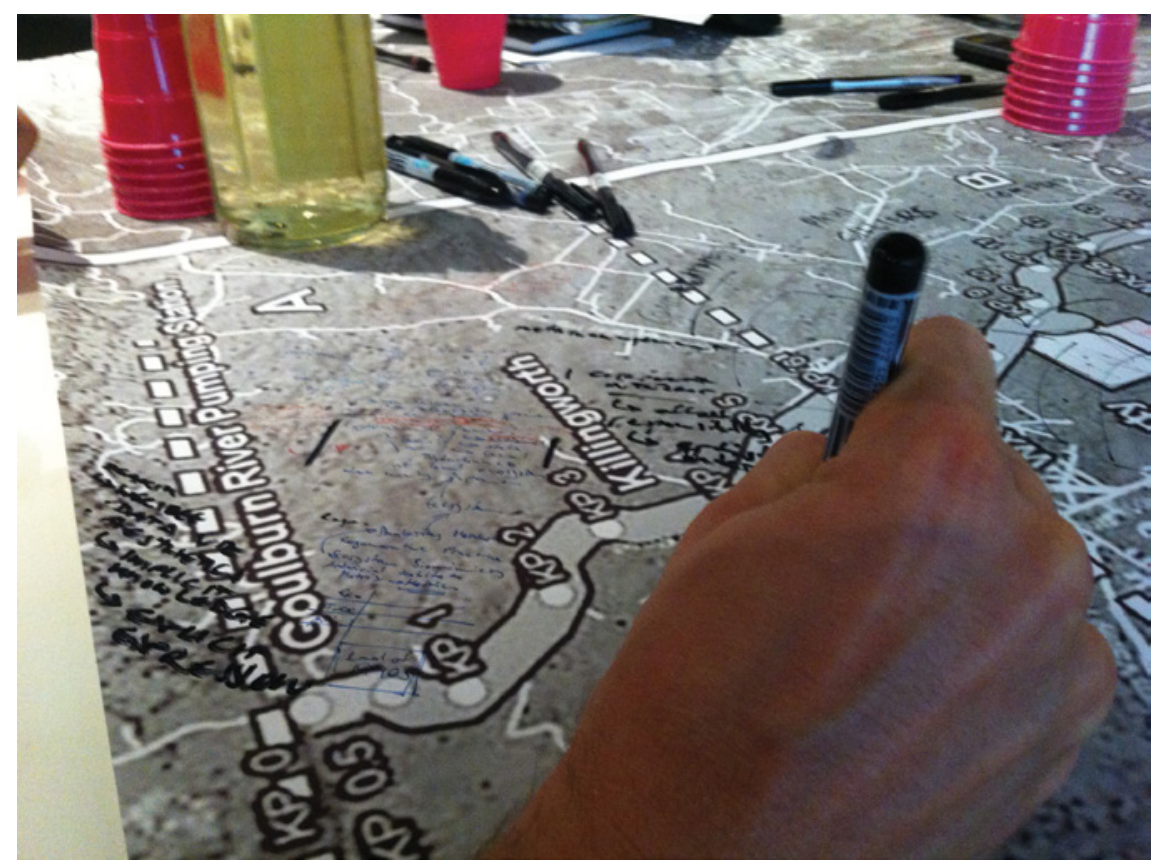

FIGURE 1 Wunderkammer

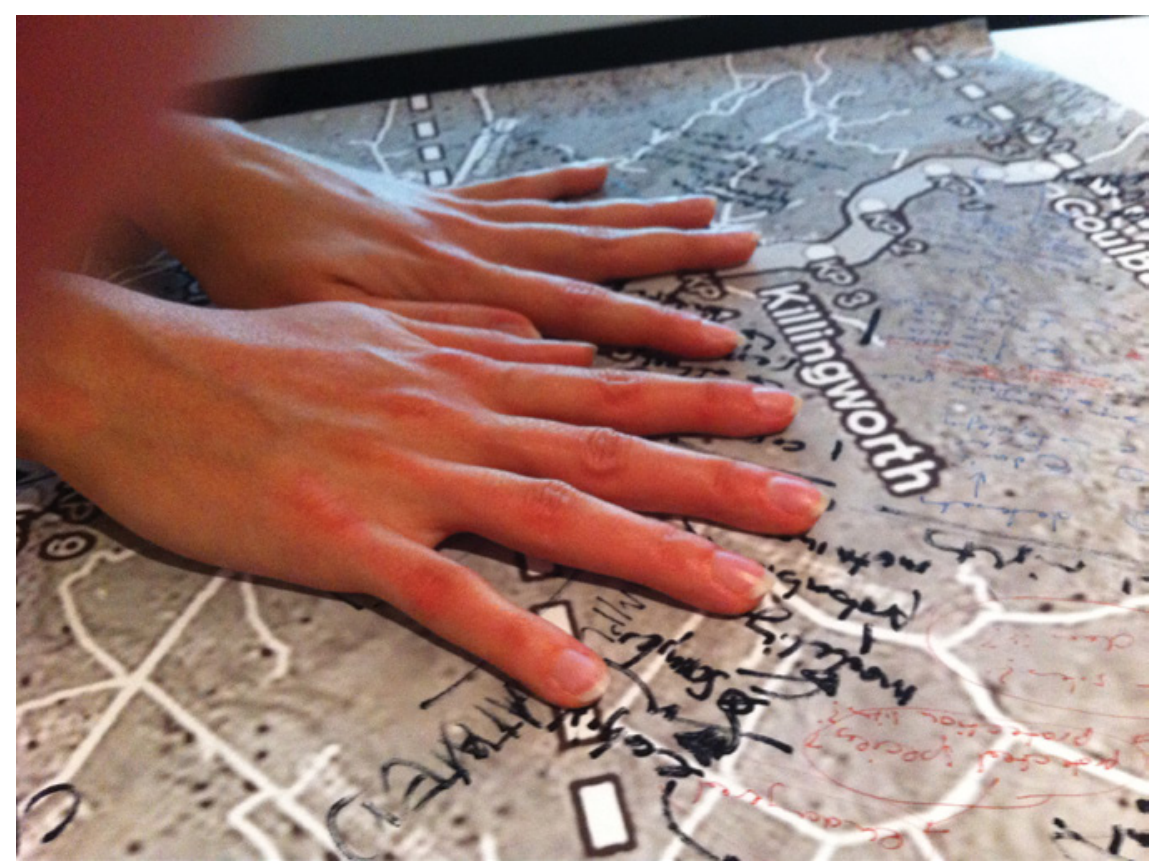

FIGURE 2 Wunderkammer 
[B] ut in a way [that's] possibly where the road stops and people were taking flight. So the road as in the capacity to use just words to where it's just like "..." and then it's like "..." - there might not be a trace of it, but as far as people's experience as to what was going on - [they were] probably the moments where everything gelled.

JAN, interview, 21 June 2017

Jan's remark left me a little discouraged. His comment confirmed what I had observed thus far through the research, and corroborated Winquist's (1982) and Manning's observations on the emergent properties of insight. It posed a substantial and additional dilemma: how to meaningfully document the felt experience of knowing?

\section{$4 \quad$ Encountering Methodological Possibilities}

The second event in the investigation, intertextual film night, originally ran from 2008-2015. It takes its name from the use of intertextual analysis as the method to connotatively respond to research inquiry. Specifically, the event uses film as a provocative lens through which issues, predominantly centred around embodiment, can be explored. In the original intertextual film night event, the discussions were framed around the relationship of disordered mental states and the body. In the case of the intertextual film night event recomposed for the $\mathrm{PhD}$, the discussion focussed on what bodily knowledge potentiates in research practice. The film selected for this event was a documentary featuring women in deeply intimate relationships with objects Married to the Eiffel Tower (Piotrowska, 2008). My intention with this screening was to use the subject matter of the film as a provocation to test ideas around how the physical self is implicated in research practice.

As indicated earlier, physicality as a site of knowing is fundamentally implicated in the Nicolescuian approach to transdisciplinary practice. For Nicolescu, this is expressed through "the Subject-Object interaction" (2010, p. 22; 2014, p.187). The transcendence of the Subject from her/his disciplinary camp is achieved through an engagement with knowing that includes "intuition, imagination ... and the body" (de Freitas, et al., 1994, para. 19). In entering into the intertextual film night event the participants were asked to explore what is implicated through the interaction of physical knowing with the research object?

1 The capitalisation of the Subject and Object here follows Nicolescu's convention of capitalizing certain terms to distinguish them as central concepts to his system. 
As the group made their way through the film, the ensuing discussions began to elaborate upon this premise further by testing ideas in which the approach to the research object is understood in terms of partnership, rather than in the terms of its application. Gerda made a reference to a competitive archer featured in the film, citing her as an example of this approach. In the film, the archer describes the intimacy she felt with her bow. She states "I ... swear that my blood just flowed from my arm and went right into him" (Piotrowska, 2008, 03:55 mark). Gerda submitted this account of the bow suggests its function in this context is not just as a tool that supports the archer's skill, but additionally as an extension of the archer's physicality, and thus, an extension of what is made possible through that assemblage.

Gerda's observation provided me with some in-roads into the challenge of documenting felt, non-linguistic moments in my audio recordings. Instead of thinking of the recordings as discrete artefacts of the transcription process, I triangulated the recordings alongside the participant interviews and field notes from a reflective journal (referred to at the time as a research 'companion'). Where possible, I corroborated what I had committed to memory against the recollection of the participants in their recorded post-event interviews. As I implemented this practice a secondary 'conversation' began to emerge. One which reconsidered the participant observations in 'partnership' with my felt experience and in dialogue with the data as it was emerging.

With this practice in place I approached the third and final transdisciplinary event, parallel fascinations. The original parallel fascinations event ran from 2014-2016 and was so called to indicate the event's intention to invite practitioners with dual practices to correspond one practice against the other. This approach was undertaken to explore what congruences might be revealed and build thresholds of relation between both practices that could then be used to expand the respective possibilities of each.

An example from past practice is provided below. An architect was invited to present on his dual practices at a Parallel Fascinations event on 24June 2016. The table compares corresponding conditions between his architectural research and his research engagement practice:

TABLE 2 Parallel fascination format

Practice

Corresponding Conditions

$1 \mathrm{PhD}$ on wind phenomena in the early (architectural) design stage

2 Research engagement

Complexity; interaction

Complexity; interaction 
It had been a custom of my practice to use the event platforms I had codeveloped to showcase the work of my peers. However, in the lead up to this event I became aware that, due to the time constraints of my existing work commitments and the specificity of the strategy underpinning this event, finding a presenter to operationalize this method would take longer than I had allowed in my research timeline. As a result, I volunteered myself the role of presenter, aiming to examine my emerging $\mathrm{PhD}$ research on transdisciplinarity against my creative practice. For the purpose of the research project, each event I had recomposed for this investigation had facilitated discussion within the group through the use of what I had been referring to as a 'conversation prompt'. Essentially, the prompts took the form of large print outs depicting a subject related to the topic under discussion. These were then spread out on the table in front of the participants and accompanied by a variety of pens. The participants were encouraged to use the prompt as a way to concretize emergent thoughts as they surfaced during the discussions.

For the earlier wunderkammer event, I printed out an enlarged map of the geographic area impacted by the environmental policies under discussion (see figure 3$)$.

The conversation prompt for the following intertextual film night event featured a print out of the different meanings implicated by the word 'practice' (see figure 4).

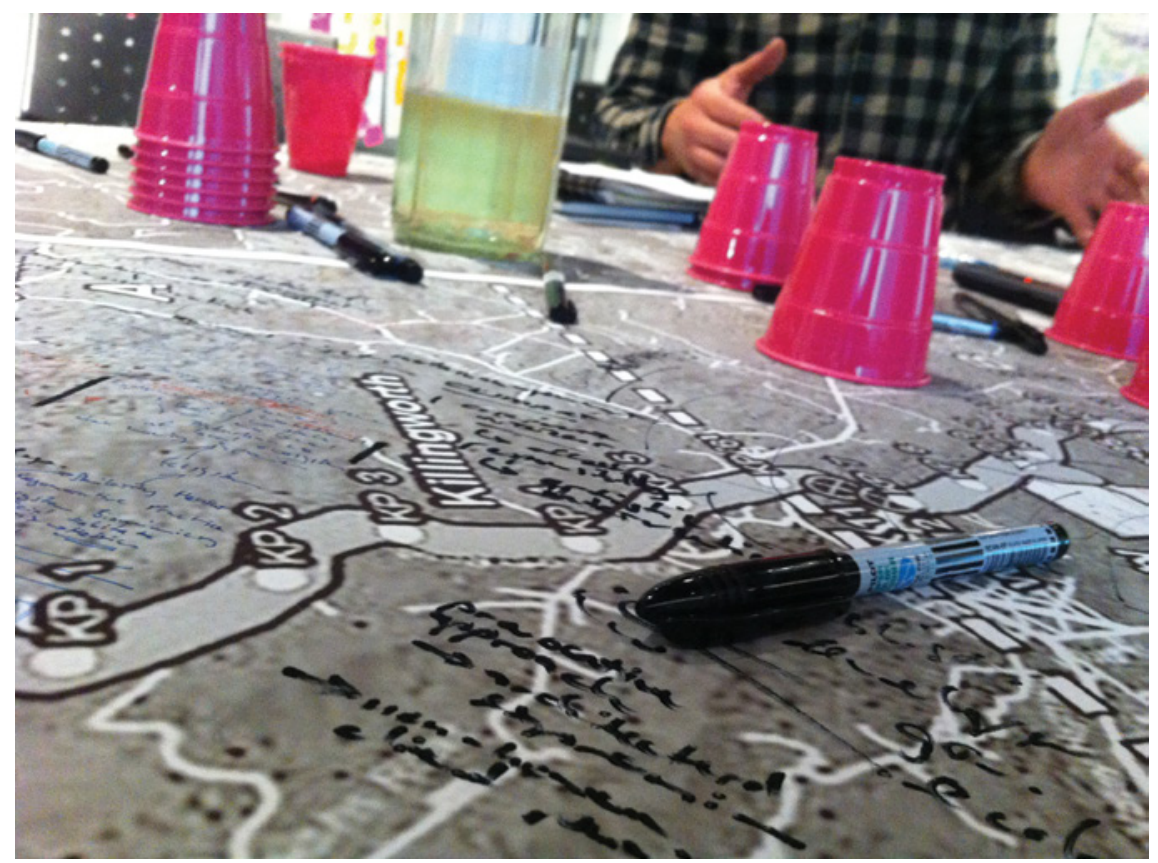

FIGURE 3 Wunderkammer 


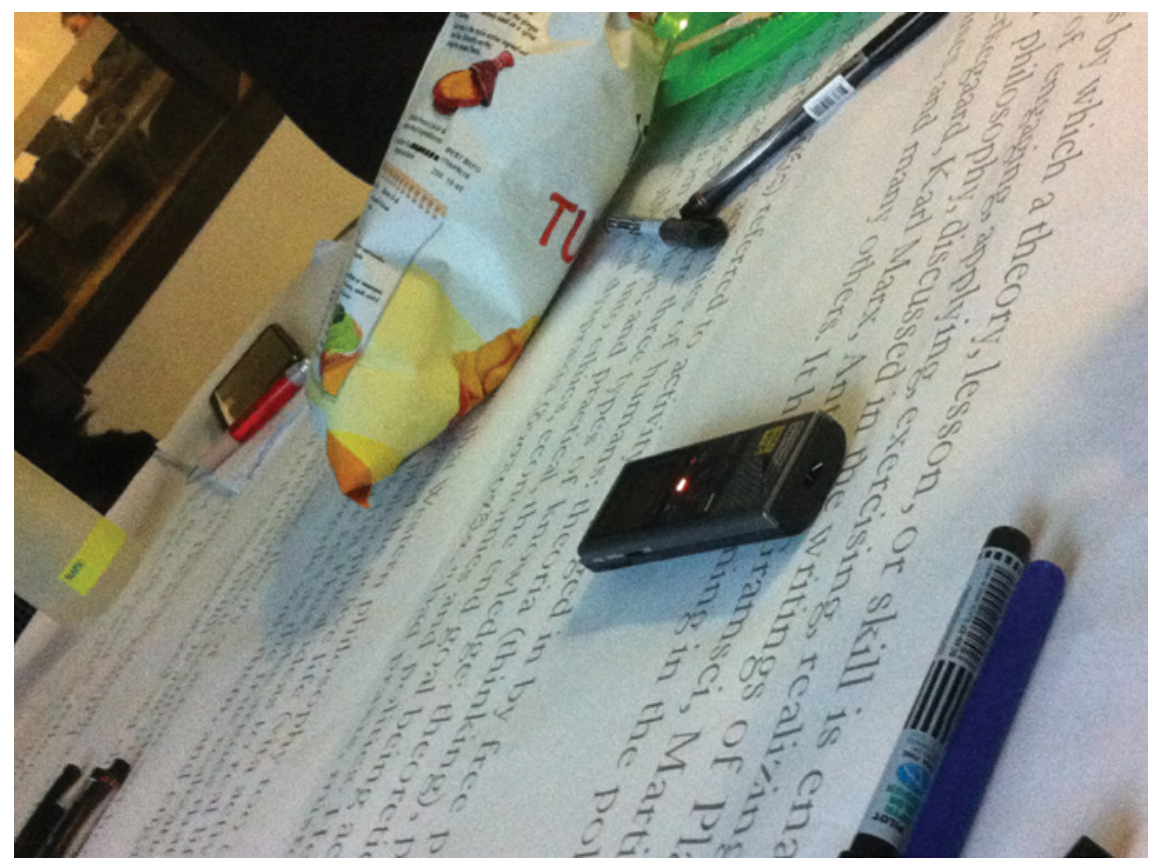

FIGURE 4 Intertextual conversation prompt

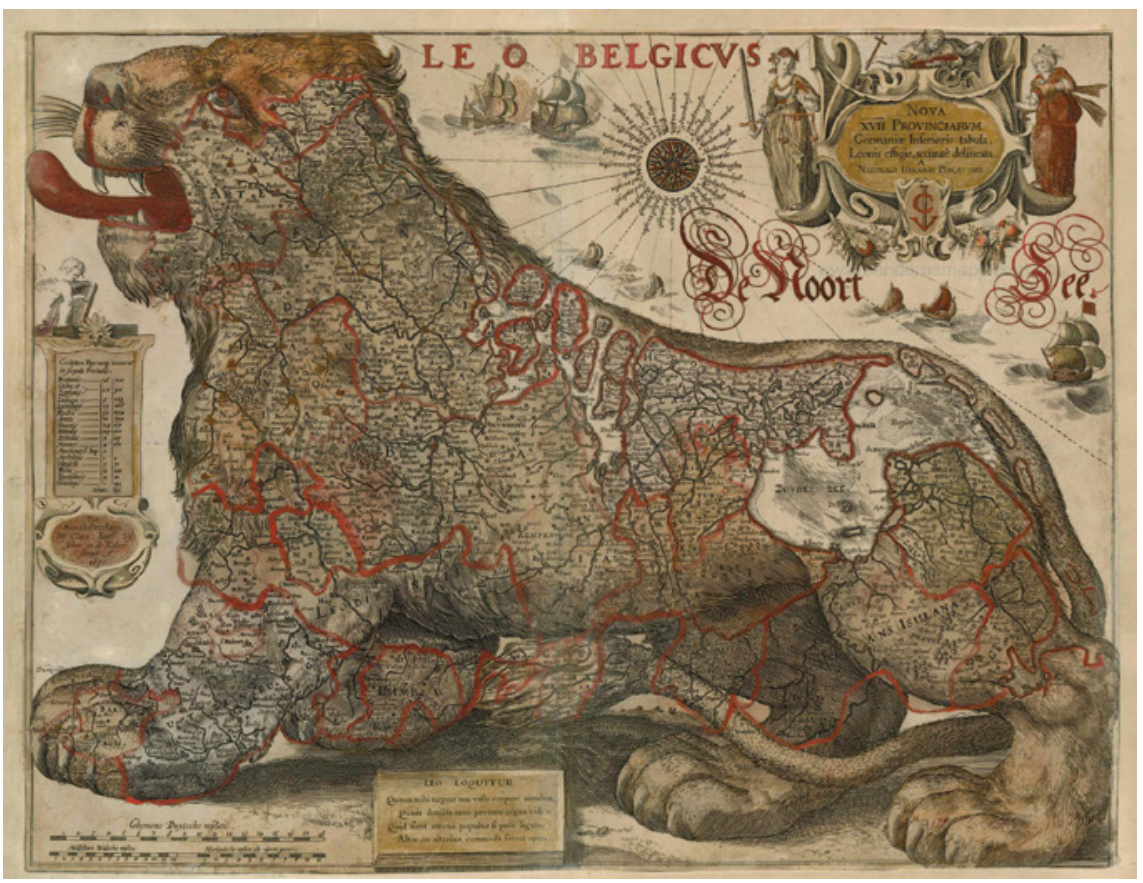

FIGURE 5 Visscher Gerritsz, Leo Belgicus 
In the case of the parallel fascinations event, the prompt was adapted from an image used in the original version of this event, a map design known as the Leo Belgicus (see figure 5).

The Leo Belgicus typically overlays a map of Belgium onto the form of a lion. The version used for the parallel fascinations event was an interpretation of a version by Jodocus Hondius (1611) done by Visscher Gerritsz (1630). My intention in choosing the Leo Belgicus map as the parallel fascinations' 'conversation prompt' was to use the map's metaphorical allusions as a provocation for discussion.

Maps are way-finding devices, both literally and metaphorically, offering a theoretical utility which extends beyond the geographical. They are analysed within the context of their relationship to power (Conley, 1998); examined in the ways in which they encode subjective feeling or experience (Conley, 1998), and inverted as a way to displace and challenge assumptions (Vaughan, 2009). Design researcher and educator Lauren Vaughan's latter proposition has been helpful in the way I have thought of, and used, maps in relation to my transdisciplinary practice. Vaughan (2009) conceives of 'lostness' as a wandering that redirects the attention and, through this process "ideally we are open to the unexpected ... [and to] the structure of another" (Vaughan, 2009, p. 3). Her description of acts of mapping that "[trace] our gaze or our finger across the surface of [the map's] representation [and] place ourselves there (literally or through association)" (Vaughan, 2009, p. 1-2), recalls the projective capacity art historian William. J. T. Mitchell (1995) makes of the connection between human visual experience and touch. Mitchell argues that:

Human visual experience from the ancient materialists through Descartes, Diderot, and Merleau-Ponty has consistently been modelled on the sense of touch, with the figure of the blind man as the central feature or 'meta-picture' of the visual process.

MITCHELL, 1995, p. 543

The idea Mitchell presents of modelling visual experience around the blind man and the sense of touch, offered a way to think through the exploration of unfamiliar territories of knowledge. It recalled to me the various discursive 


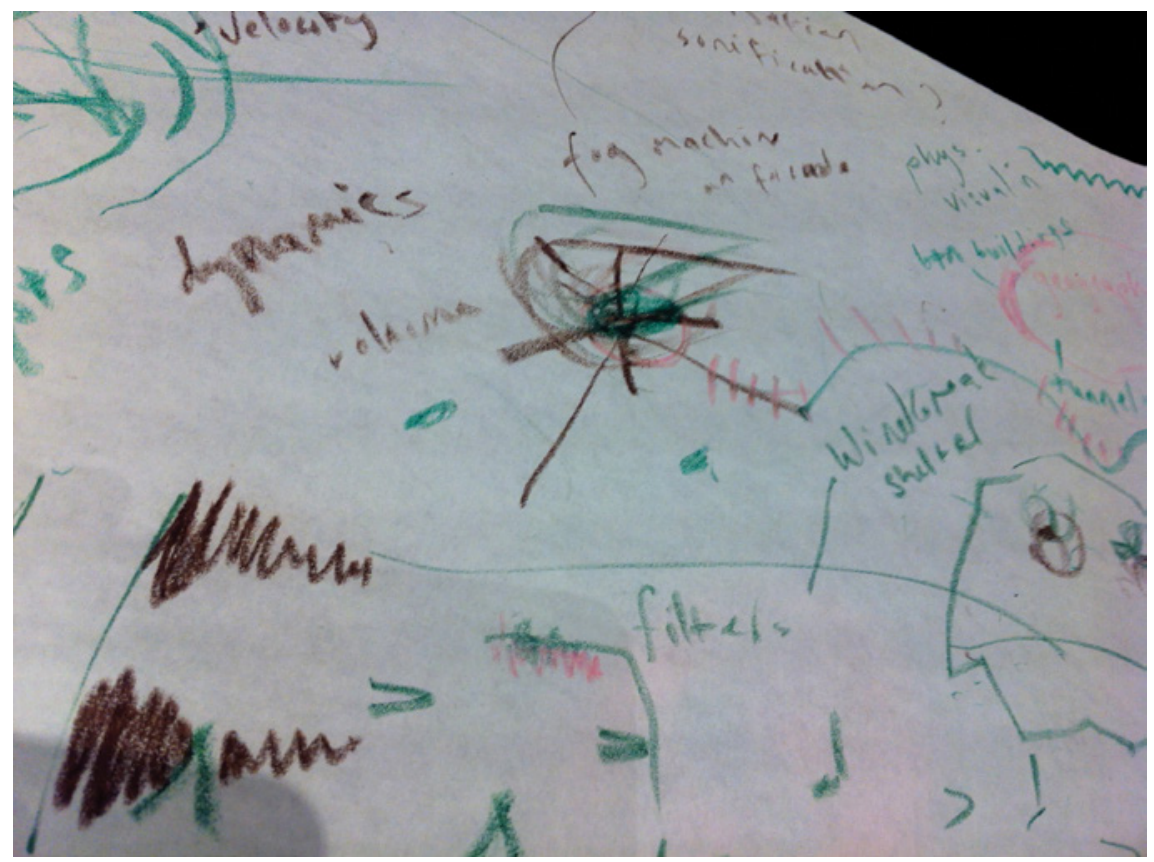

FIGURE 6 Parallel fascinations: participant notes figure 6

passes I had observed in my practice of groups tracing the longitudes and latitudes of new ideas in order to make sense of its 'form'. This idea returns me to early observations of a participant's notes in the original parallel fascinations events (see figure 6). Her sketches appeared less as self-commentary and more a form of mapping in the style of the French Flâneurs, observing points of connection rather than plotting a set direction.

I began the presentation for parallel fascinations with a brief explanation of the event's history and its intent. This was followed by a similarly short description of my creative practice, in which I attempted to speculatively correspond the role metaphor served in my creative practice with its function in my transdisciplinary research. Throughout this discussion I displayed the most recent work my creative practice had produced, all of which referenced mythological figures. I explained to the group that my aim when producing 


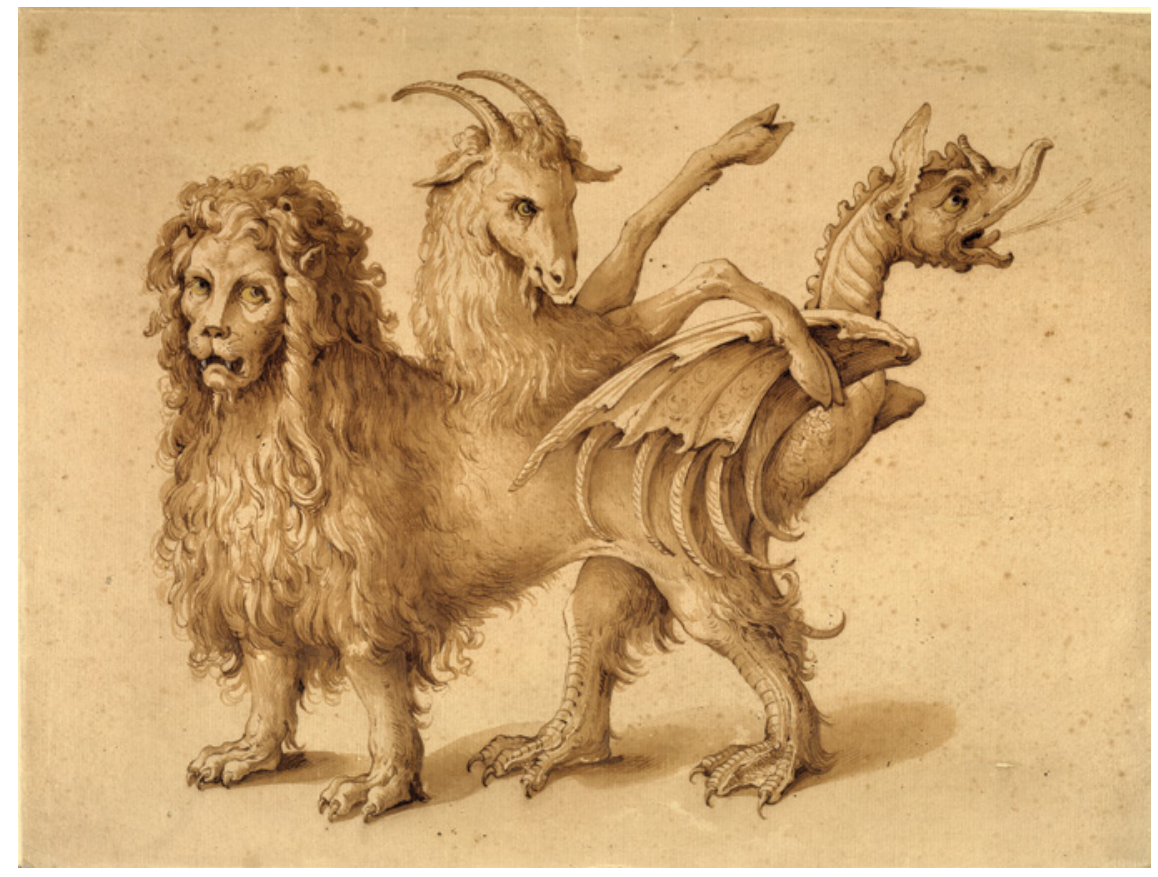

FIGURE 7 Jacopo Ligozzi, Chimera (sense 1)

these works was to create intense moments of felt experience for its audience. It was an attempt on my part to recreate the sensations I experienced when viewing the work of the painter Francis Bacon or installation artist Mike Nelson, a response I felt had been otherwise absent in the experience I had with digitally mediated work at that time. From that explanation I moved to points of connection I felt might be emerging between Jacopo Ligozzi's chimera (1590-1610) (see figure 7) which I had been using as a metaphorical reference to transdisciplinarity with the mythological figures of my creative practice.

Around mid-point through these discussions, the participant Gerda drew my attention to one of the creative works I had produced which featured a digitally projected and animated gargoyle in relation to the map of the Leo Belgicus used as the conversation prompt. She observed correspondences in the characteristics of both, suggesting that:

The operations of your practice] kind of [perform an] affective metaphorical way of grafting on ... [and] completing space in a way that was imagined ... Which is what this [Leo Belgicus] does ... it ... is an imaginary 
representation of space - and that's what you ... do [in your practice] as well.

GERDA, interview, 27 October 2017

I was floored by Gerda's comment. It was an operation in my creative practice I had never been cognisant of before, but once pointed out was so conspicuous I felt foolish for not noticing it sooner. Evident in both were imagined representations of space. However, in the case of the Leo Belgicus these spatial conditions were confined to the materiality of the medium. By contrast, the gargoyle, through the act of digital projection, was able to breach the limit of materiality and inhabit any environment into which it was cast. It was with this new awareness, of breaching the limits of materiality through digital projection, that I began the process of transcribing the audio recording for the parallel fascinations event.

Concurrent to my transcription work was a presentation I was developing for a review of my research. As I worked on both I found the transcription/ coding process spilling over into the presentation slides I was putting together for the review. Initially I had set out to code the transcriptions around participant feedback on the creative research methods in my practice, but found

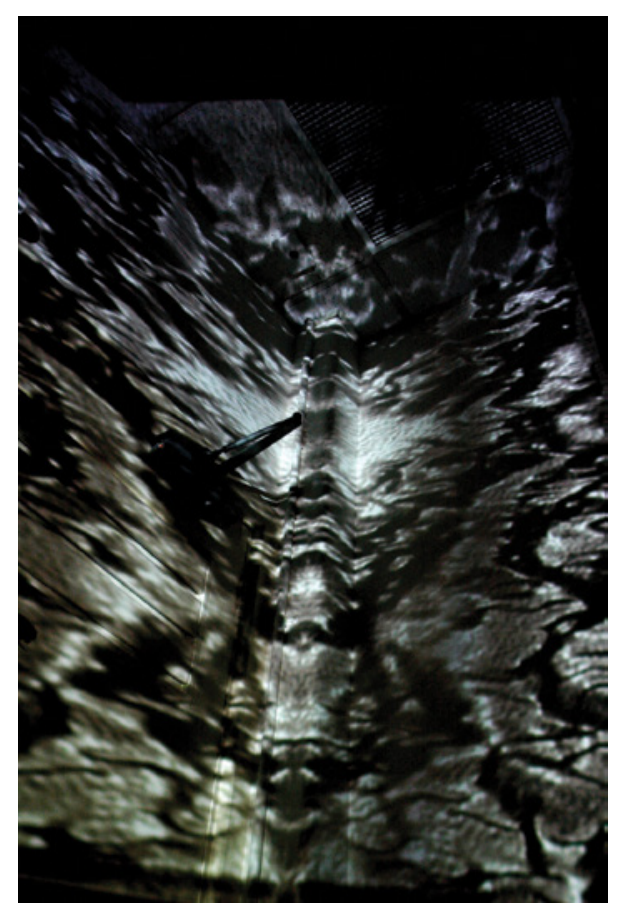

FIGURE 8

Romaine Logere, gargoyle 
instead themes emerging which addressed research practice itself (both mine and the participants) in addition to the felt experience of ideation.

I found myself drafting long horizontal lines within the slides to indicate the event's duration. Running down from that line were further vertical lines representing each moment as it occurred in the encounter. For moments within the conversation considered less significant to the research inquiry, shorter lines at greater distances apart were rendered into the diagram. These were contrasted by longer lines in closer proximity, which I used to represent the nonverbal sounds of movement indicating a gathering of group momentum. Moments of particular insight were timestamped into the diagrams based on the point at which they appeared in the discussion transcripts.

I found the experience of attempting this visual transcription of the encounters paralleled the labours of the written one; both cycling variously through moments from the mundane to enlightening. With each stroke of a line rendered I was reminded too of my early drawing practice, and the movement of the hand during the cross-hatching process. Connecting the experience of working in a qualitative way with my creative research training allowed a further realisation into how embedded my creative practice is in my understandings and approach to transdisciplinarity, and made sense of the diagrammatic artefacts that were emerging as products of the research. The diagrams were tools emerging through and supportive of the coding process. I began to think of these artefacts as diagrams of intensity. Registering moments of indwelling in the transdisciplinary encounter in which insight is shared and extends the participant's received set of concepts. The diagrams recalled to me the Deleuzian construct of the diagram as a "map of sensation ... and force ... whose lines [are] registers of experience" (Vellodi, 2018, p. 304).

In the context of this practice, the diagrams of intensity sought to transcribe the felt experience of transdisciplinary indwelling into a visual artefact that could be used for analysis.

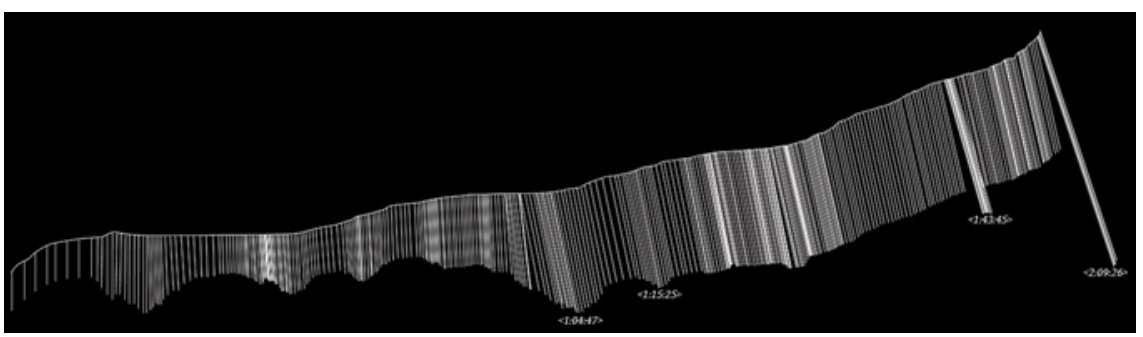

FIGURE 9 Wunderkammer diagram 


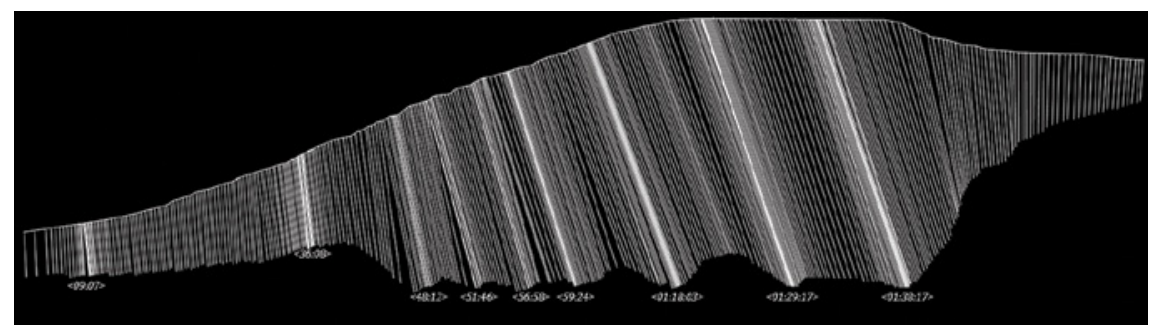

FIGURE 10 Intertextual film night diagram

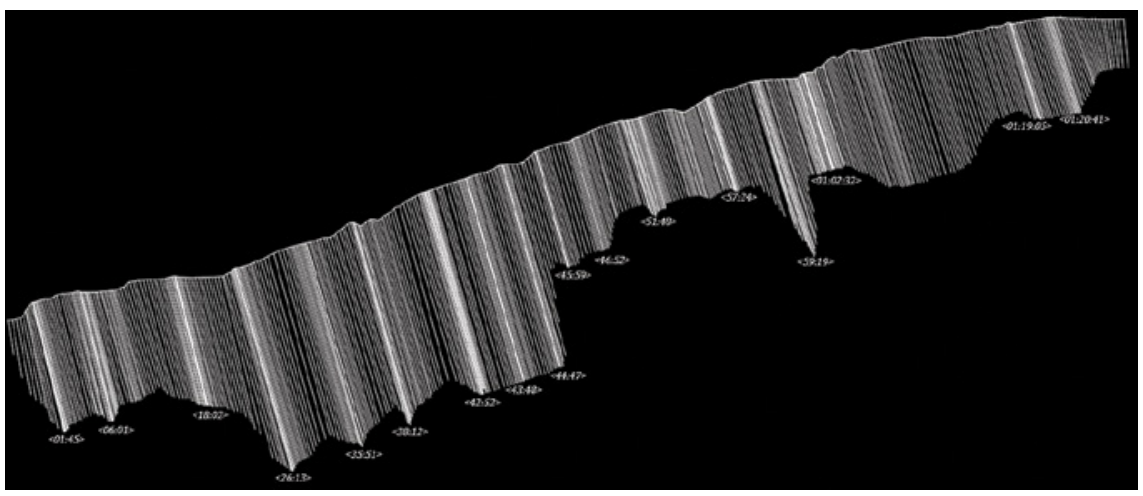

FIGURE 11 Parallel fascinations diagram

Once completed I was able to use the diagrams of intensity to track the group's momentum in each event, gain valuable information into the utility of each event's production, and insight into some of the significant felt moments of the research. The diagrams were able to clearly demonstrate that the group momentum had a temporal aspect. As the event discussions progressed through the investigation, I was able to identify an exponential increase of collective momentum within the group based on the increased frequency of the timestamped insights. Four occurrences appeared in the wunderkammer diagram, which then increased to nine in the intertextual film night diagram, and seventeen in the following parallel fascinations diagram. This provided a vision into the practice which corroborated my earlier observations around increased knowledge exchange in relation to increased group affinity but had been previously unable to verify.

Earlier in this article I referred to a steadily increasing body of work concerning the operation of physical knowing in research. Through the investigation of my transdisciplinary practice I have found myself repeatedly speculating why this approach is not more widely discussed within the literature. 
As I have indicated, while the role and value of bodily knowing is acknowledged (de Freitas, et al., 1994; McGregor, 2015; Nicolescu, 2010, 2014; Winquist, 1982) less attention is given to evidencing it and its contributions. At its core this article asks: If bodily knowledge is implicated in the Subject's transdisciplinary transcendence (Nicolescu, 2010, 2014) and is correlated to the ideational incubation process of indwelling (Winquist, 1982) in what ways can we attend to this process in practice? Certainly, the language used around the role of bodily knowing in research - that it offers transcendence (Nicolescu, 2010, 2014), revelatory Aha! moments (Winquist, 1982) and radical shifts (Manning, n.d.) - suggests it is a significant actor in altering what is possible. While further examination is called for, the investigation of my own transdisciplinary practice supports these indications. It additionally offers a visualization method to capture felt data in a way that permits its analysis, and hopefully contributes a broader utility to others faced with similar methodological challenges.

\section{Acknowledgements}

I wish to thank the anonymous reviewers whose critical reading was instrumental in improving and clarifying this manuscript.

\section{References}

Aguilar, J. L. (1981). Insider research: an ethnography of a debate, D. A. Messerschmidt (Ed.), Anthropologists at home in North America: Methods and issues in the study of one's own society, 185-201, New York, US: Cambridge University Press.

de Freitas, L., Morin, E. and Nicolescu, B., (1994). The Charter Of Transdisciplinarity | Inters.Org. [online] Inters.org. Retrieved from: http://www.inters.org/Freitas-Morin -Nicolescu-Transdisciplinarity.

Barbour, K. (2004). Embodied ways of knowing. Waikato Journal of Education, 10, 227-238.

Barnacle, R. (2009). Gut Instinct: The body and learning. Educational Philosophy and Theory, 41(1), 22-33. https://www.doi.org/10.1111/j.1469-5812.2008.00473.x.

Brenner, J. E. (2004). Logic, art and transdisciplinarity: A new logic for the new reality. Technoetic Arts, 1(3), 169-180.

Chong, K. H. (2008). Coping with Conflict, Confronting Resistance: Fieldwork Emotions and Identity Management in a South Korean Evangelical Community. Qualitative Inquiry, 369-39o. https://www.doi.org/10.1007/s11133-008-9114-0. 
Conley, T. (1998). Mapping in the Folds: Deleuze "Cartographe", Discourse, 2o(3), $123-138$.

Lexico (2020). Lexico. [online]. Retrieved from: https://www.lexico.com/definition/ emissary.

Manning, E. (n.d.). Interview with Erin Manning, on SenseLab [video]. Retrieved from http://www.senselab.ca/wp2/tangent.

McGregor, S. L. T. (2015). Transdisciplinary Knowledge Creation, P. Gibbs (Ed.), Transdisciplinary Professional Learning and Practice, 9-24, Cham, CH: Springer.

Mitchell, W. J. T. (1995). Interdisciplinarity and Visual Culture. Art Bulletin, 77(4), 540-544.

Nicolescu, B. (2010). Methodology of Transdisciplinarity-Levels of Reality, Logic of the Included Middle and Complexity. Transdisciplinary Journal of Engineering \& Science, 1(1), 19-38. https://www.doi.org/10.22545/2010/0009.

Nicolescu, B. (2014). Methodology of Transdisciplinarity. World Futures, 7o(3), 186-199. https://www.doi.org/10.1080/02604027.2014.934631.

Pillow, W. S. (2003). Confession, catharsis, or cure? Rethinking the uses of reflexivity as methodological power in qualitative research. International Journal of Qualitative Studies in Education, 16(2), 175-196. https://www.doi.org/10.1080/095183903200006 0635 .

Piotrowska, A. (Producer \& Director). (2008). Married to the Eiffel Tower [Documentary]. United Kingdom: Blink Films.

St. Pierre, E. A. (1997). Methodology in the fold and the irruption of transgressive data. International Journal of Qualitative Studies in Education, 10(2), 175-189. https://www .doi.org/10.1080/095183997237278.

Taylor, J. (2011). The intimate insider: negotiating the ethics of friendship when doing insider research. Qualitative Research, $11(1), 3^{-22}$.

Vaughan, L. (2009). The art of being lost: An alternate approach to mapping. In: Proceedings of the 24th International Cartographic Conference, Santiago, Chile, 15-21 November, 2009.

Vellodi, K. (2018). Diagram: Deleuze's augmentation of a topical notion, Word \& Image, 34(4), 299-309. https://www.doi.org/10.1080/02666286.2018.1473145.

Winquist, R. W. (1982). What are transdisciplinary principles? Issues in Integrative Studies, $67(1), 49-67$. 